\title{
Aligned Horizontal Silica Nanochannels by Oxidative Self-Sealing of Patterned Silicon Wafers
}

Xin Chen, ${ }^{*}$ Ran Ji, ${ }^{\S}$ Martin Steinhart, Alexey Milenin, Kornelius Nielsch, Ulrich Gösele Max Planck Institute of Microstructure Physics, Weinberg 2, D-06120 Halle/Saale, Germany

Fax: (+49)-345-5511223

E-mail: xchen@mpi-halle.de 


\section{Oxidative self-sealing of grooves in a patterned (100)-oriented silicon-on-insulator (SOI) wafer}

Oxidative self-sealing is a versatile approach that can be applied to a broad range of different wafer architectures, as outlined in Fig. S1. For example, we patterned (100)oriented silicon-on-insulator (SOI) wafers (SOITEC, France) containing an intermediate $250 \mathrm{~nm}$ thick $\mathrm{SiO}_{2}$ layer (“insulator") covered by a $500 \mathrm{~nm}$ thick Si layer (SOI layer) and an uppermost $50 \mathrm{~nm}$ thick $\mathrm{SiO}_{2}$ layer (Fig. S1a) by LIL. We removed residual resist from the developed areas with $\mathrm{O}_{2}$ plasma using the same procedure as in the case of the (110) wafers described in the main article. Subsequently, we transferred the line pattern generated by LIL into the uppermost $\mathrm{SiO}_{2}$ layer with $\mathrm{CHF}_{3}$ gas plasma $(100 \mathrm{~W}, 20 \mathrm{sccm}$, 10 mbar for 5 minutes). During this etching step, the remaining resist on the wafer served as a mask, and grooves formed where LIL had removed it. Finally, we etched Si selective to $\mathrm{SiO}_{2}$ with $\mathrm{Cl}_{2}$ plasma using an Oxford PlasmaLab 100 system in an inductively coupled plasma (ICP) mode at room temperature $(50 / 1200 \mathrm{~W}, 100 \mathrm{sccm}$, and $26.6 \mathrm{mbar}$ for 2.5 minutes). As a result, the lithographically defined line pattern was transferred into the SOI layer, whereas the underlying $\mathrm{SiO}_{2}$ "insulator" layer remained unpatterned (Fig. S1b). We oxidized the wafers thus treated in air to seal the grooves in the SOI layer (Fig. S1c).

Fig. S2 shows a SEM image of a patterned SOI wafer prior to the oxidation. The grooves had a width of $180 \mathrm{~nm}$ at the top, of $120 \mathrm{~nm}$ at the bottom, and a depth of $550 \mathrm{~nm}$. The center-to-center distance amounted to $410 \mathrm{~nm}$, and the walls between two adjacent grooves had a thickness of $230 \mathrm{~nm}$ at the top, and of $290 \mathrm{~nm}$ at the bottom. A SEM image of a sample kept at $950^{\circ} \mathrm{C}$ for $2 \mathrm{~h}$ is seen in Fig. S2b. The Si appears in dark contrast and 
the $\mathrm{SiO}_{2}$ layer is the light grey area. $\mathrm{A} \mathrm{SiO}_{2}$ shell surrounds the cores of the walls between adjacent grooves, which still consist of $\mathrm{Si}$. The sealing is not complete at this stage, but the differences in the thickness of the oxide layer are clearly discernible. At the bottom, the diameter of the remaining hollow space is larger than above, and the oxide is thinner. The retarded oxidation at the concave corners at the bottom of the grooves clearly causes this morphology. The self-sealing of the grooves is completed if the samples are oxidized at $1000^{\circ} \mathrm{C}$ for $180 \mathrm{~min}$ (Fig. S2c,d). As a result, we obtained an array of aligned, horizontal nanochannels with a center-to-center distance of $\sim 400 \mathrm{~nm}$, which is in line with the period introduced by LIL (Fig. S2a). As in the case of the (110) wafers, they exhibit triangular cross sections, having one edge with a length of $\sim 50 \mathrm{~nm}$ oriented parallel to the initial $\mathrm{Si} / \mathrm{SiO}_{2}$ interface. Their height amounts to $\sim 60 \mathrm{~nm}$ (Fig. S2d).

It is obvious that the dimensions of the nanochannels can be adjusted by the conditions under which LIL and thermal oxidation are performed. However, only a post-oxidative etching step with hydrofluoric acid (HF) allows widening the channels. We immersed a wafer as that seen in Fig. S2c and d containing horizontal nanochannels into a 5 wt- $\%$ aqueous $\mathrm{HF}$ solution for 3 minutes at room temperature to remove the $\mathrm{SiO}_{2}$ formed during the first oxidation step. The period of the pattern in the wafer remained unaltered, but the thickness of the Si walls between the grooves decreased to $130 \mathrm{~nm}$. Subsequently, we sealed the grooves by oxidizing the wafer again in air at $1000^{\circ} \mathrm{C}$ for $4 \mathrm{~h}$. The horizontal nanochannels thus obtained exhibited again triangular cross sections. The edge length increased to $\sim 240 \mathrm{~nm}$, and the height of the channels to $300 \mathrm{~nm}$ (Fig. S3). 


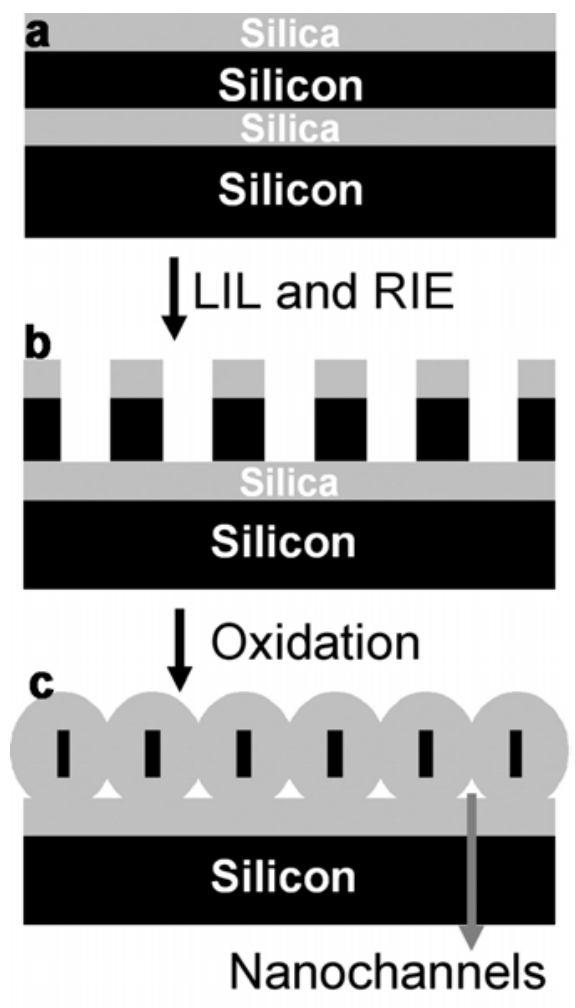

Figure S1. Schematic diagram of the fabrication of aligned, horizontal nanochannels in a (110)-oriented Si wafer containing nano-grooves by oxidative self-sealing of the grooves. Because of the retardation of the oxide growth upon thermal oxidation at the concave corners, hollow nanochannels are still present at the bottom of the grooves, whereas above the bottoms the oxide layers growing from the opposite walls of the grooves impinge. The light grey areas denote $\mathrm{SiO}_{2}$, the black areas $\mathrm{Si}$. 


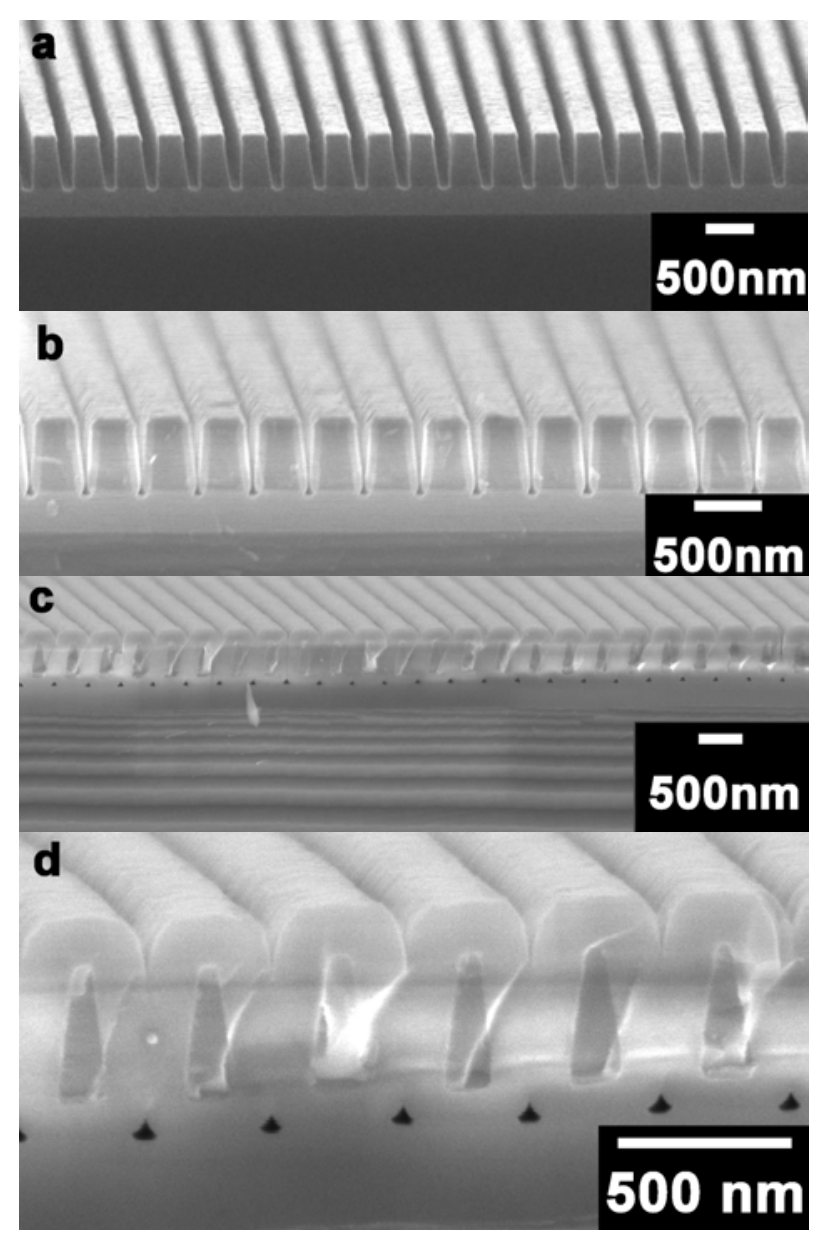

Figure S2. SEM images of cross-sections of (100)-oriented SOI wafers at different stages of the self-sealing procedure. (a) Wafer containing nano-grooves prior to the oxidation; (b) wafer oxidized for $2 \mathrm{~h}$ at $950^{\circ} \mathrm{C}$; (c, d) wafer oxidized at $1050^{\circ} \mathrm{C}$ for $180 \mathrm{~min}$. The light grey areas are the $\mathrm{SiO}_{2}$ layer, whereas the remaining $\mathrm{Si}$ appears in darker contrast. The nanochannels appear as dark spots in the $\mathrm{SiO}_{2}$ layer. (c) Overview; (d) Detail. 


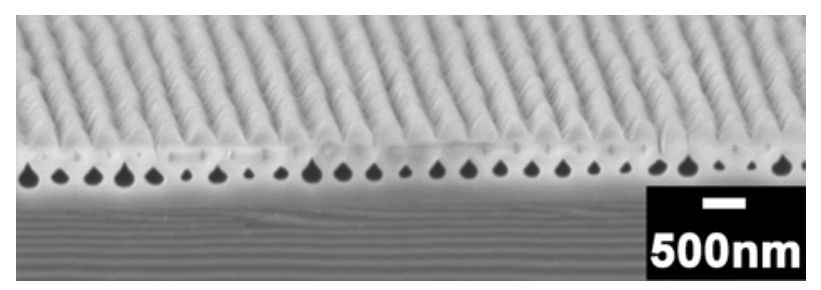

Figure S3. SEM image of a cross-section of a (100)-oriented SOI wafer prepared as that seen in Fig. S2c and d, whose nanochannels had been widened by performing postoxidative etching with an aqueous HF solution and a subsequent second oxidation step at $1000^{\circ} \mathrm{C}$ for $240 \mathrm{~min}$. 